\title{
An Update on What is Happening in Orthopaedics in 2018: A Look Into the Future of Orthopaedics
}

Prosthetics is making rather rapid advances; the use of electronic control of the prosthetic arm or leg with the use of signals from muscles at the level of amputations is hoped to provide better control of the prosthesis making the lighter and easier to control. The advent of the micro systems has enabled this development and the day when a bionic arm or leg would be available is not far off.

The advancements in the use of biologics to control and maybe even cure the various forms of arthritis would enable the saving of joints and patients would be able to continue functioning with their own joints would be an asset. The advent of gene manipulations and the ability to replace or correction of the gene responsible would change the outlook on the way one looks at arthritis and complete cures would be common place happening. The development of gene manipulation/substitution would also change the outlook and treatment of various malignancies. The survival rate in osteosarcoma could be construed as an example where the five year survival has been pushed up to around $50 \%$ from the very short survivals of yesteryear.

The miniaturization of the various components of various parts has improved prosthetics to enable them to be lighter, smaller, more accessible and hopefully if they can be motorized the functional aspects would improve exponentially.

The improvements in metallurgy to make composites stronger, lighter, more biocompatible would allow the developments of more ways to provide medications as depots to be used instead of daily administration especially in locations of poor accessibility. These would also allow the manufacture of appliances (i.e.) joints that would hopefully outlive the patients need for them and also allow better modes of fixation (biologic versus artificial modes).

I would say that the future of orthopaedics is bright. In spite of the countless challenges, which this surgical field has faced, orthopaedic surgeons will continue to provide prime quality treatments.

We will continue to have the satisfaction and deep meaning gained from contributing to the quality of life of our patients and their maximum musculoskeletal potential.

We are in a profession that will have increasing demands for our services and an exciting evolving future. We need to be involved in shaping it for our patients and our profession.

Cite this Guest Comment as:

Anathakrishnan CV. An Update on what is happening in

Orthopaedics in 2018: A look into the future of Orthopaedics. Int

Healthcare Res J 2018; 2(2):24. doi: 10.26440/IHRJ/02_02/161

Dr. C.V. Ananthakrishnan

MD (Orthopaedics)

Senior Joint Replacement Surgeon

Ex Clinical Associate Professor (Dept. of Orthopaedic Surgery)

Texas Tech University, School of Medicine

Lubbock, Texas, USA

Ex-Medical Director, St Mary of the Plains Hospital

Texas, USA 